\title{
Hidden Charcot-Marie-Tooth 1A as Revealed by Peripheral Nerve Imaging
}

\author{
Kazumoto Shibuya, Toshiki Yoshida, Sonoko Misawa, Yukari Sekiguchi, Minako Beppu, \\ Hiroshi Amino, Yo-ichi Suzuki, Tomoki Suichi, Atsuko Tsuneyama, \\ Keigo Nakamura and Satoshi Kuwabara
}

\begin{abstract}
:
Peripheral nerve imaging techniques have recently increasingly revealed their usefulness. We herein describe a man who had a subacute progression of symptom, diffuse and prominent proximal demyelination and conduction block, suggesting a diagnosis of inflammatory demyelinating polyneuropathy. Additional nerve imaging techniques revealed homogeneous and prominent nerve hypertrophy without proximal accentuation and the findings implied inherited polyneuropathies. Intravenous immunoglobulin was administered, and both the symptoms of weakness and findings of nerve conduction studies (NCS) improved. Subsequent genetic testing unveiled Charcot-Marie-Tooth 1A. To diagnose peripheral nerve disorders, a careful history, physical examination and NCS are essential diagnostic tools, but the findings of this case suggest the importance of nerve imaging techniques in clinical situations.
\end{abstract}

Key words: Charcot-Marie-Tooth 1A, chronic inflammatory demyelinating polyneuropathy, ultrasonography, magnetic resonance neurography, nerve enlargement, cross-sectional areas

(Intern Med 58: 3157-3161, 2019)

(DOI: 10.2169/internalmedicine.3040-19)

\section{Introduction}

Peripheral nerve imaging techniques, such as ultrasonography and magnetic resonance neurography (MRN), have increasingly revealed their usefulness. In peripheral nerve disorders, while a nerve conduction study is the standard tool to assess nerve functions, mainly in the distal and intermediate portions of the peripheral nerves, nerve imaging techniques can depict nerve morphology from the nerve roots to distal portions. Nerve enlargement, disclosed by ultrasonography and MRN, has been reported in peripheral nerve diseases, including chronic inflammatory demyelinating polyneuropathy (CIDP) and Charcot-Marie-Tooth (CMT) (1-4). In general, prominent, homogeneous and smooth nerve enlargement, from proximal to distal symptom, has been identified in inherited demyelinating polyneuropathies, such as CMT type 1 , but moderate and inhomogeneous enlargement with proximal accentuation has been re- ported in CIDP.

CMT1A patients occasionally have an inflammatory component. While the prevalence of an inflammatory neuropathy in CMT is 1 in 250, the chance of CIDP in the general population is $1-5$ per 100,000 (5). The prevalence of inflammatory polyneuropathy in CMT has been assumed to be related to genetic backgrounds and a myelin abnormality. Briefly, hereditary neurodegenerative disorders frequently involve an inflammatory component, and/or abnormal myelin may be vulnerable to autoimmune mechanisms (5). However, an appropriate diagnosis for such patients could be difficult, because both demyelinating CMT and CIDP share some common neurophysiological findings.

We herein describe a patient with "hidden" CMT whose diagnosis was supported by nerve imaging techniques, as well as electrophysiologic and genetic testing. This patient's case suggests the importance of nerve imaging techniques in clinical situations. 
Table 1. Nerve Conduction Study and Cross-sectional Areas Measured by Ultrasonography.

\begin{tabular}{|c|c|c|c|c|c|c|c|c|}
\hline & \multicolumn{6}{|c|}{ Nerve conduction study } & \multicolumn{2}{|c|}{$\begin{array}{l}\text { Nerve cross-sectional } \\
\text { area }\left(\mathrm{mm}^{2}\right)\end{array}$} \\
\hline & $\begin{array}{l}\text { Latency } \\
(\mathrm{ms})\end{array}$ & $\begin{array}{c}\text { CMAP } \\
\text { amplitude } \\
(\mathrm{mV})\end{array}$ & Velocity (m/s) & $\begin{array}{c}\text { F-wave } \\
\text { latency } \\
(\mathrm{ms})\end{array}$ & $\begin{array}{c}\text { SNAP } \\
\text { amplitude } \\
(\mu \mathrm{V})\end{array}$ & $\begin{array}{l}\text { Velocity } \\
(\mathrm{m} / \mathrm{s})\end{array}$ & Patient & Normal \\
\hline \multicolumn{9}{|l|}{ Median nerve } \\
\hline Wrist & 9.4 & 3.7 & & 73.5 & 2 & 17 & 17 & $8(2)$ \\
\hline Forearm & & & & & & & 21 & $6(2)$ \\
\hline Elbow & 21.6 & 2.2 & 18 (Forearm) & & & & 30 & $5(1)$ \\
\hline Proximal Arm & 23.9 & 2.5 & 24 (Proximal arm) & & & & 31 & $6(1)$ \\
\hline \multicolumn{9}{|l|}{ Ulnar nerve } \\
\hline Wrist & 9.0 & 3.3 & & 79.0 & N.E. & N.E. & 16 & $4(1)$ \\
\hline Forearm & & & & & & & 14 & $4(1)$ \\
\hline Below elbow & 18.9 & 2.4 & 21 (Forearm) & & & & & \\
\hline Above elbow & 23.7 & 1.0 & 11 (Across elbow) & & & & 26 & $4(1)$ \\
\hline Proximal Arm & 28.1 & 0.9 & 28 (Proximal am) & & & & 21 & $4(1)$ \\
\hline \multicolumn{9}{|l|}{ Tibial nerve } \\
\hline Ankle & N.E. & N.E. & N.E. & N.E. & & & & \\
\hline \multicolumn{9}{|l|}{ Sural nerve } \\
\hline Lower leg & & & & & N.E. & N.E. & & \\
\hline
\end{tabular}

Normal data are given as the mean (SD).

CMAP: compound muscle action potential, SNAP: sensory nerve action potential

\section{Case Report}

A 35-year-old man, who felt numbness for 8 months and weakness for 2 months in his feet, was admitted to our hospital. He had normal growth and did not have any preceding infection, other past medical history or any family history of neurological diseases, but he had run slow from his childhood. A neurological examination disclosed distal dominant and symmetric weakness with grade 4 on the Medical Research Council scale, muscle atrophy in the hands and feet with pes cavus, absent tendon reflexes, hypoesthesia in the feet and a steppage gait.

A blood analysis revealed normal findings, including negative results for antinuclear antibody, anti-human immunodeficiency virus antibody, syphilis antibody and Mprotein, and a normal thyroid function, vitamin B1 and B12 concentrations, and angiotensin converting enzyme level. Cerebrospinal fluid (CSF) showed prominent albuminocytologic dissociation, with a protein level of $117 \mathrm{mg} / \mathrm{dL}$. Nerve conduction studies in the right extremities showed prolonged distal latencies, decreased nerve conduction velocities, saliently prolonged F-wave latencies and a decreased compound muscle action potential (CMAP) and sensory nerve action potential amplitudes, suggesting diffusely distributed and prominent proximal demyelination (Table 1). Especially, conduction block across the elbow was found in the ulnar nerve (Table 1, Fig. 1A).

A subacute progression of symptom, diffuse and prominent proximal demyelination and conduction block at the elbow indicated a diagnosis of inflammatory polyneuropathy, but an additional MRN study revealed homogeneous and smooth nerve hypertrophy without proximal accentuation, utilizing short tau inversion recovery images (Fig. 1B) $(6,7)$. Moreover, ultrasonography was performed on the right median and ulnar nerves at the wrist, midforearm, elbow and mid-arm and disclosed homogeneous and prominent nerve hypertrophy with about 3-4 time enlargement (Fig. 1C, D and Fig. 2), compared to those of 15 healthy subjects (Table 1). Especially, the nerve crosssectional area in the ulnar nerve at the elbow was larger than at other sites, without any dislocation of the ulnar nerve. These imaging findings implied inherited polyneuropathy, and distal dominant weakness, muscle atrophy, pes cavus, and prominently decreased conduction velocities were reaffirmed (1-3, 7-11). Intravenous immunoglobulin (0.4 $\mathrm{g} / \mathrm{kg}$ for consecutive 5 days) was administered, and both the feelings of weakness and abnormal CMAP amplitude reduction significantly improved (Table 2 and Fig. 1A). Subsequently, genetic testing unveiled peripheral myelin protein 22 gene duplication on chromosome 17p11.2-12, which was considered to be evidence of CMT type 1A. He was therefore diagnosed as having CMT1A with an inflammatory component.

\section{Discussion}

In the present case, nerve imaging findings, as measured by ultrasonography and MRN, suggested inherited polyneuropathy and also supported the discovery of hidden CMT1A. One previous study examined ultrasonographic differences between inherited and acquired demyelinating polyneuropathies and identified prominent (more than 3 times the normal level) and diffuse (at the mid-humerus and forearm in 
A

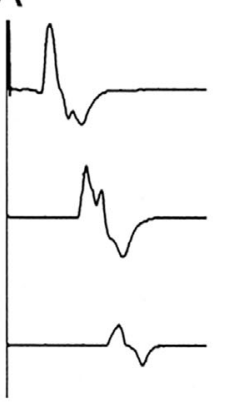

Before treatment

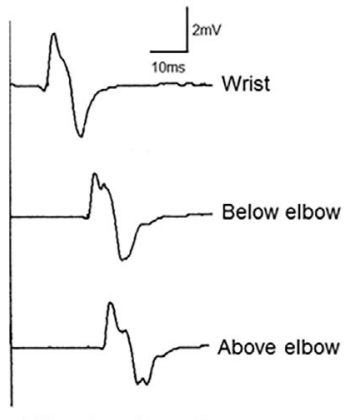

After treatment
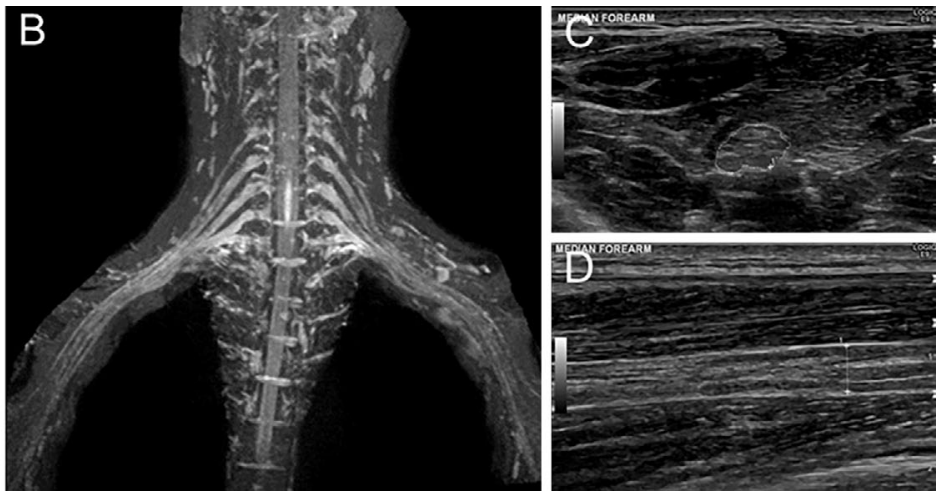

Figure 1. Nerve conduction study, magnetic resonance neurography and ultrasonographic images in a patient with hidden Charcot-Marie-Tooth 1A. (A) A motor nerve conduction study was performed in the ulnar nerve at the wrist, below elbow and above elbow. Diffuse slowing with an abnormal amplitude reduction across the elbow was disclosed before treatment. This abnormal amplitude reduction was confirmed by a sufficient strength stimulation, $100 \mathrm{~mA}$ and $0.2 \mathrm{~ms}$, and the patient recovered after treatment. These findings imply inflammatory neuropathy. (B) Magnetic resonance neurography revealed homogeneous and smooth nerve enlargement, without proximal accentuation. (C) Ultrasonography was performed in the median nerve at the forearm and depicted an enlarged nerve cross-sectional area, with $21 \mathrm{~mm}^{2}$ (3.5 times larger than normal). (D) The longitudinal scan in the median nerve at the forearm disclosed a smooth surface. These imaging findings suggest inherited neuropathy.
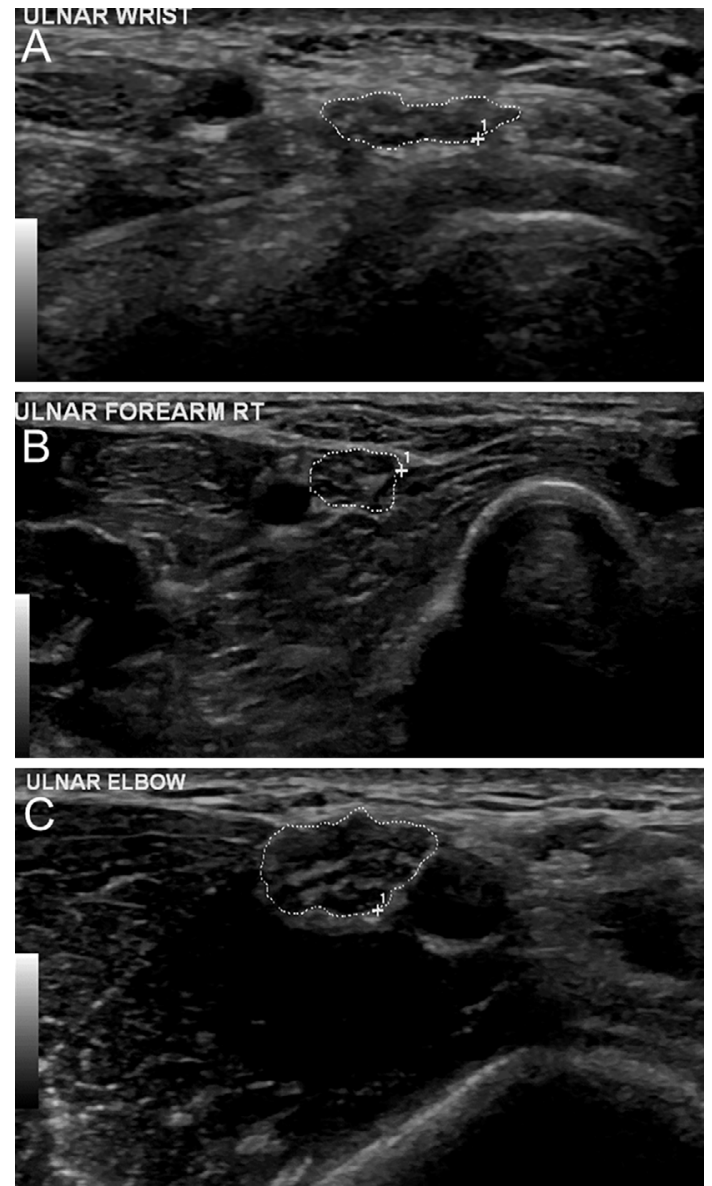

Figure 2. Ultrasonographic images of the ulnar nerve in a patient with hidden Charcot-Marie-Tooth 1A. Ultrasonography discloses an enlarged ulnar nerve at the wrist (A), forearm (B) and elbow (C) with 16, 14 and $26 \mathrm{~mm}^{2}$ cross-sectional areas, respectively. These studies suggest nerve enlargement. median and ulnar nerves) enlargement in CMT1 (3). Another study investigated nerve sizes of the median and ulnar nerves and nerve roots at 6 sites for each in demyelinating CMT and CIDP and found that nerve sizes in CMT were greater at all sites than CIDP (2). Furthermore, the number of enlargement sites made it easy to differentiate CMT from CIDP, with high sensitivity and specificity (over 90\%, respectively), suggesting a diffuse enlargement in CMT, but not in CIDP. This diffuse enlargement was also found in another study, and the homogeneity enlargement score also differentiated CMT from immune-mediated demyelinating neuropathies (12). Additionally, previous MRN studies found a smooth and diffuse enlargement without any proximal accentuation in CMT1A $(1,13)$. Taken together, prominent, homogenous and smooth nerve enlargement, without proximal accentuation, thus suggesting the presence of CMT. The diagnosis of this case was supported by the nerve imaging findings.

Previous studies have described that elevated CSF protein, cut-off $>100 \mathrm{mg} / \mathrm{dL}$, suggests inflammatory neuropathy, but some articles have reported cases of CMT1A with extremely increased CSF protein levels, from 100 to $300 \mathrm{mg} /$ $\mathrm{dL}$ (14-16). Leakage from enlarged nerve roots and partial block of CSF circulation are assumed to increase proteins in those cases. In the present case, the CSF protein level might have increased due to both an inflammatory component and nerve hypertrophy.

This report is associated with some limitations. If we measure nerve cross-sectional areas, utilizing ultrasonography, at more than 4 sites and apply the established scoring system for evaluating nerve enlargement, then we may be able to clearly identify hidden CMT1A (2-4). Moreover, if 
Table 2. Nerve Conduction Study Findings after Treatment.

\begin{tabular}{|c|c|c|c|c|c|c|}
\hline & $\begin{array}{l}\text { Latency } \\
\text { (ms) }\end{array}$ & $\begin{array}{c}\text { CMAP } \\
\text { amplitude } \\
(\mathrm{mV})\end{array}$ & Velocity $(\mathrm{m} / \mathrm{s})$ & $\begin{array}{c}\text { F-wave } \\
\text { latency } \\
(\mathrm{ms})\end{array}$ & $\begin{array}{c}\text { SNAP } \\
\text { amplitude } \\
(\mu \mathrm{V})\end{array}$ & $\begin{array}{c}\text { Velocity } \\
(\mathrm{m} / \mathrm{s})\end{array}$ \\
\hline \multicolumn{7}{|l|}{ Median nerve } \\
\hline Wrist & 12.4 & 3.7 & & 73.7 & N.E. & N.E. \\
\hline \multicolumn{7}{|l|}{ Forearm } \\
\hline Elbow & 23.8 & 3.3 & 22 (Forearm) & & & \\
\hline Proximal Arm & 27.8 & 3.3 & 18 (Proximal arm) & & & \\
\hline \multicolumn{7}{|l|}{ Ulnar nerve } \\
\hline Wrist & 7.6 & 2.4 & & 77.2 & 3 & 11 \\
\hline \multicolumn{7}{|l|}{ Forearm } \\
\hline Below elbow & 18.3 & 2.1 & 20 (Forearm) & & & \\
\hline Above elbow & 23.0 & 2.1 & 11 (Across elbow) & & & \\
\hline Proximal Arm & 26.8 & 2.1 & 24 (Proximal am) & & & \\
\hline \multicolumn{7}{|l|}{ Tibial nerve } \\
\hline Ankle & N.E. & N.E. & N.E. & N.E. & & \\
\hline \multicolumn{7}{|l|}{ Sural nerve } \\
\hline Lower leg & & & & & N.E. & N.E. \\
\hline
\end{tabular}

CMAP: compound muscle action potential, SNAP: sensory nerve action potential

we have additional supportive data, such as nerve biopsy findings, gadolinium enhancement in MRN, nerve conduction findings at another side and ultrasonography for lower limbs, it might also be possible to identify insidious inflammation which would thus help to made an accurate diagnosis. However, in the present case, ultrasonography at 4 sites in the upper limb and MRN provided us with satisfactory evidence to identify hidden CMT1A.

To diagnose peripheral nerve disorders, a careful history, physical examination and nerve conduction studies are essential tools and very important, but nerve imaging techniques may be an additional helpful tool in complicated cases. If CMT patients experience a subacute deterioration, then treatment for any coexistent inflammatory component should be considered. Conversely, if inflammatory polyneuropathy patients have neuroimaging findings suggestive of CMT1A, then genetic testing should also be considered.

The authors state that they have no Conflict of Interest (COI).

\section{Financial Support}

Drs. Shibuya, Misawa, Sekiguchi, Beppu and Kuwabara received research support from the Ministry of Education, Culture, Sports, Science, and Technology of Japan. Dr. Kuwabara received research support from the Ministry of Education, Culture, Sports, Science, and Technology of Japan, and Grants-in-Aid from the Research Committee of CNS Degenerative Diseases, the Ministry of Health, Labor and Welfare of Japan.

\section{References}

1. Shibuya K, Sugiyama A, Ito $S$, et al. Reconstruction magnetic resonance neurography in chronic inflammatory demyelinating polyneuropathy. Ann Neurol 77: 333-337, 2015.

2. Sugimoto T, Ochi K, Hosomi N, et al. Ultrasonographic nerve en- largement of the median and ulnar nerves and the cervical nerve roots in patients with demyelinating Charcot-Marie-Tooth disease: distinction from patients with chronic inflammatory demyelinating polyneuropathy. J Neurol 260: 2580-2587, 2013.

3. Zaidman CM, Harms MB, Pestronk A. Ultrasound of inherited vs. acquired demyelinating polyneuropathies. J Neurol 260: 3115 3121, 2013.

4. Grimm A, Rattay TW, Winter N, Axer H. Peripheral nerve ultrasound scoring systems: benchmarking and comparative analysis. J Neurol 264: 243-253, 2017.

5. Ginsberg L, Malik O, Kenton AR, et al. Coexistent hereditary and inflammatory neuropathy. Brain 127: 193-202, 2004.

6. Attarian S, Azulay JP, Boucraut J, Escande N, Pouget J. Terminal latency index and modified $\mathrm{F}$ ratio in distinction of chronic demyelinating neuropathies. Clin Neurophysiol 112: 457-463, 2001.

7. Kuwabara S, Ogawara K, Misawa S, Mori M, Hattori T. Distribution patterns of demyelination correlate with clinical profiles in chronic inflammatory demyelinating polyneuropathy. J Neurol, Neurosurg, Psychiatry 72: 37-42, 2002.

8. Decard BF, Pham M, Grimm A. Ultrasound and MRI of nerves for monitoring disease activity and treatment effects in chronic dysimmune neuropathies - Current concepts and future directions. Clin Neurophysiol 129: 155-167, 2018.

9. Krajewski KM, Lewis RA, Fuerst DR, et al. Neurological dysfunction and axonal degeneration in Charcot-Marie-Tooth disease type 1A. Brain 123: 1516-1527, 2000.

10. Kuwabara S, Isose S, Mori M, et al. Different electrophysiological profiles and treatment response in 'typical' and 'atypical' chronic inflammatory demyelinating polyneuropathy. J Neurol, Neurosurg, Psychiatry 86: 1054-1059, 2015.

11. Mathey EK, Park SB, Hughes RA, et al. Chronic inflammatory demyelinating polyradiculoneuropathy: from pathology to phenotype. J Neurol, Neurosurg, Psychiatry 86: 973-985, 2015.

12. Grimm A, Vittore D, Schubert V, et al. Ultrasound pattern sum score, homogeneity score and regional nerve enlargement index for differentiation of demyelinating inflammatory and hereditary neuropathies. Clin Neurophysiol 127: 2618-2624, 2016.

13. Khadilkar SV, Yadav RS, Soni G. A practical approach to enlargement of nerves, plexuses and roots. Pract Neurol 15: 105-115, 2015.

14. Ishigami N, Kondo M, Nakagawa M. [Case of Charcot-Marie- 
Tooth disease type 1A with increased cerebrospinal fluid proteins and nerve root hypertrophy]. Rinsho Shinkeigaku 48: 419-421, 2008 (in Japanese, Abstract in English).

15. Rajabally YA, Adams D, Latour P, Attarian S. Hereditary and inflammatory neuropathies: a review of reported associations, mimics and misdiagnoses. J Neurol, Neurosurg, Psychiatry 87: 10511060, 2016.

16. Pareyson D, Testa D, Morbin M, et al. Does CMT1A homozy- gosity cause more severe disease with root hypertrophy and higher CSF proteins? Neurology 60: 1721-1722, 2003.

The Internal Medicine is an Open Access journal distributed under the Creative Commons Attribution-NonCommercial-NoDerivatives 4.0 International License. To view the details of this license, please visit (https://creativecommons.org/licenses/ by-nc-nd/4.0/).

(C) 2019 The Japanese Society of Internal Medicine Intern Med 58: 3157-3161, 2019 